\title{
Uniform High-Order Difference Schemes for a Singularly Perturbed Two-Point Boundary Value Problem
}

\author{
By Eugene C. Gartland, Jr.
}

\begin{abstract}
A family of uniformly accurate finite-difference schemes for the model problem $-\varepsilon u^{\prime \prime}+a(x) u^{\prime}+b(x) u=f(x)$ is constructed using a general finite-difference framework of Lynch and Rice [Math. Comp., v. 34, 1980, pp. 333-372] and Doedel [SIAM J. Numer. Anal., v. 15, 1978, pp. 450-465]. A scheme of order $h^{p}$ (uniform in $\varepsilon$ ) is constructed to be exact on a collection of functions of the type $\left\{1, x, \ldots, x^{p}\right.$, exp $\left.\left(\frac{1}{\varepsilon}\right\} a\right)$, $\left.x \exp \left(\frac{1}{\varepsilon} \int a\right), \ldots, x^{p-1} \exp \left(\frac{1}{\varepsilon} \int a\right)\right\}$. The high order is achieved by using extra evaluations of the source term $f$. The details of the construction of such a scheme (for general $p$ ) and a complete discretization error analysis, which uses the stability results of Niederdrenk and Yserentant [Numer. Math., v. 41, 1983, pp. 223-253], are given. Numerical experiments exhibiting uniform orders $h^{p}, p=1,2,3$, and 4, are presented.
\end{abstract}

1. Introduction. We are concerned with the numerical approximation by finite-difference techniques of the linear two-point boundary value problem

$$
\begin{gathered}
L u \equiv-\varepsilon u^{\prime \prime}+a(x) u^{\prime}+b(x) u=f(x), \quad 0<x<1, \\
u(0)=g_{0}, \quad u(1)=g_{1} .
\end{gathered}
$$

Such model problems are studied because, among other reasons, they describe (when $\varepsilon$ is small compared to $a$ ) physical situations in which convective forces dominate diffusive forces. This circumstance arises often in fluid flow and convective heat transport problems.

It is assumed, at a minimum, that $a, b$, and $f$ are bounded continuous functions and that $a(x) \geqslant \underline{a}>0$ on $[0,1]$. This last condition prohibits the development of turning points on interior layers (see, for example, [27]). Greater smoothness conditions are required in some of the theorems that follow.

The numerical approximation of model problems like (1.1) above has been the object of investigation of numerous researchers for some time. Stiff vector systems have been attacked by finite differences in [1], [18], [19], and [34], by collocation methods in [4] and [5], and by asymptotic/numerical techniques in [15] and [16] (in relation to this last approach, see also [9] for an application to a single nonlinear equation). The single nonlinear equation similar to (1.1) has been approached by integrating to steady state the related time-dependent problem using one-sided differences of the Engquist-Osher type [2], [26], and [30]. The linear model problem (1.1) and its analogues in conservative form have been approximated numerically

Received March 29, 1985; revised April 21, 1986.

1980 Mathematics Subject Classification. Primary 65L10; Secondary 34E15. 
using various projection methods including collocation [14] and Galerkin techniques [6], [28], [29], and [33].

The present paper is concerned with finite-difference approximations, which have received their share of attention (see, for example, [7], [8], [13], [17], and [20] and references contained therein). A continuous source of analyses of various approaches to these problems is supplied by the proceedings of the BAIL conferences edited by J. J. H. Miller [22], [23], and [24]. We also mention the book [12].

It is the object of the present paper to develop and analyze a general approach to constructing uniform (with respect to $\varepsilon$ ) high-order finite-difference approximations to (1.1). The technique utilizes the HODIE framework of Lynch and Rice [21] and Doedel [11]. It proceeds by constructing a difference approximation locally that is exact on a collection of functions of the type

$$
\begin{aligned}
\left\{1, x, \ldots, x^{K},\right. & \exp \left(\frac{1}{\varepsilon} \int a(x) d x\right), \\
& \left.x \exp \left(\frac{1}{\varepsilon} \int a(x) d x\right), \ldots, x^{L} \exp \left(\frac{1}{\varepsilon} \int a(x) d x\right)\right\} .
\end{aligned}
$$

The procedure is automated in the sense that the difference coefficients are computed numerically, by solving a small local linear algebraic system, rather than evaluated from closed form expressions. Any uniform order can be achieved, in theory, and numerical experiments illustrating uniform convergence rates up to $O\left(h^{4}\right)$ are presented.

The high order is achieved by using extra evaluations of the differential operator $L$, that is, of the coefficient functions $a$ and $b$, and of the source term $f$. The theory is developed under the assumption that $\int a(x) d x$ and $a^{\prime}(x)$ are known, but it is then shown that using discrete approximations to these, built on the same evaluations of $a(x)$ as are required by the basic finite-difference construction, yields the same uniform order of convergence.

The truncation error analysis utilizes a decomposition, proved in Section 2, of the true solution

$$
u(x ; \varepsilon)=A(x ; \varepsilon)+B(x ; \varepsilon) \exp \left(-\frac{1}{\varepsilon} \int_{x}^{1} a\right),
$$

where, for sufficiently smooth data, the functions $A$ and $B$ have continuous derivatives up to a given finite order that can be bounded independently of $\varepsilon$. This decomposition implies many a priori estimates on $u$ and its derivatives that have appeared elsewhere in the literature, and it does not rely on monotonicity arguments and their consequent $b(x) \geqslant 0$ constraint. The discretization error analysis is accomplished using the general stability results of Niederdrenk and Yserentant [25].

2. Mathematical Preliminaries. Under the minimum assumptions above (continuity of $a$ and $b$ and positivity of $a$ on $[0,1])$, the boundary value problem (1.1) is stable, uniformly in $\varepsilon$ for all $\varepsilon$ sufficiently small. In particular, we have the following result.

THEOREM 2.1. Let the coefficient functions $a$ and $b$ of the differential operator $L$ in (1.1) be continuous on $[0,1]$, and let a satisfy $a(x) \geqslant \underline{a}>0,0 \leqslant x \leqslant 1$. Then for $\varepsilon$ 
sufficiently small, there exists a constant $C$ independent of $\varepsilon$ such that $\|v\|_{\infty} \leqslant$ $C\left\{\|L v\|_{1}+|v(0)|+|v(1)|\right\}$ for all $v \in C^{2}[0,1]$.

Proof. It follows from Theorems 9 and 10 of [25] that it is sufficient to establish that for all $\varepsilon$ sufficiently small there exists a constant $C^{\prime}$ independent of $\varepsilon$ such that all solutions $w \in C^{2}[0,1]$ of the homogeneous equation $L w=0,0<x<1$, satisfy

$$
\|w\|_{\infty} \leqslant C^{\prime}(|w(0)|+|w(1)|)
$$

But this is an immediate consequence of the standard singular perturbation construction as in [27, Section 3.1].

The stability estimate above will be used in the discretization error analyses of Section 5; the discrete approximation to (1.1) will inherit an analogous property. For the purpose of the truncation error analyses of Section 4 , we also require the following representation result about the true solution $u$ of (1.1).

THEOREM 2.2. Let $k$ be a positive integer. Then for $\varepsilon$ sufficiently small and $a, b$, and $f$ sufficiently smooth, the solution $u$ of (1.1) admits the representation

$$
u(x ; \varepsilon)=A(x ; \varepsilon)+B(x ; \varepsilon) \exp \left(-\frac{1}{\varepsilon} \int_{x}^{1} a\right),
$$

where $A$ and $B$ and their derivatives up to order $k$ can be bounded on $[0,1]$ independently of $\varepsilon$.

Proof. First express $u$ as a sum $u=v+w$, where $v$ and $w$ satisfy

$$
\begin{gathered}
-\varepsilon v^{\prime \prime}+a(x) v^{\prime}+b(x) v=f(x), \quad 0<x<1, \\
v(0 ; \varepsilon)=g_{0}
\end{gathered}
$$

and

$$
\begin{gathered}
-\varepsilon w^{\prime \prime}+a(x) w^{\prime}+b(x) w=0, \quad 0<x<1, \\
w(0 ; \varepsilon)=0, \quad w(1 ; \varepsilon)=g_{1}-v(1 ; \varepsilon) .
\end{gathered}
$$

We consider the $v$ function first. Express $v$ in the form of a perturbation expansion

$$
v(x ; \varepsilon)=v_{0}(x)+v_{1}(x) \cdot \varepsilon+\cdots+v_{k-1}(x) \cdot \varepsilon^{k-1}+V_{k}(x ; \varepsilon) \cdot \varepsilon^{k},
$$

where the coefficient functions $v_{0}, \ldots, v_{k-1}$ satisfy

$$
a(x) v_{0}^{\prime}+b(x) v_{0}=f(x), \quad v_{0}(0)=g_{0},
$$

and

$$
a(x) v_{j}^{\prime}+b(x) v_{j}=v_{j-1}^{\prime \prime}, \quad v_{j}(0)=0, \quad j=1, \ldots, k-1,
$$

and the remainder function $V_{k}$ is required to satisfy

$$
\begin{gathered}
-\varepsilon V_{k}^{\prime \prime}+a(x) V_{k}^{\prime}+b(x) V_{k}=v_{k-1}^{\prime \prime}(x), \quad 0<x<1, \\
V_{k}(0)=V_{k}(1)=0 .
\end{gathered}
$$

Now $v_{0}, \ldots, v_{k-1}$ are independent of $\varepsilon$ and can be made as smooth as we wish by making $a, b$, and $f$ sufficiently smooth. It is enough, then, to show that $\left\|V_{k}^{(p)}\right\|_{\infty}=$ $\mathcal{O}\left(\varepsilon^{-p}\right), p=0, \ldots, k$. We do this by using an integral equation formulation as in [10] and [32]. Multiplying both sides of (2.2) by $\frac{1}{\varepsilon} \exp \left(\frac{1}{\varepsilon} \int_{x}^{1} a\right)$, which is an integrating 
factor for $V_{k}^{\prime \prime}-(a(x) / \varepsilon) V_{k}^{\prime}$, and integrating from $x$ to 1 yields

$$
\begin{aligned}
V_{k}^{\prime}(x)= & V_{k}^{\prime}(1) e^{-(1 / \varepsilon) \int_{x}^{1} a}-\frac{1}{\varepsilon} \int_{x}^{1} b(y) e^{(1 / \varepsilon) \int_{y}^{x} a} V_{k}(y) d y \\
& +\frac{1}{\varepsilon} \int_{x}^{1} e^{(1 / \varepsilon) \int_{y}^{x} a} v_{k-1}^{\prime \prime}(y) d y .
\end{aligned}
$$

Our estimates on the derivatives of $V_{k}$ will follow from this, once we have control of $V_{k}^{\prime}(1)$.

To get an expression for $V_{k}^{\prime}(1)$, integrate (2.3) from $x$ to 1 and use the boundary condition $V_{k}(1)=0$ to get

$$
\begin{aligned}
V_{k}(x)= & -V_{k}^{\prime}(1) \int_{x}^{1} e^{-(1 / \varepsilon) \int_{y}^{1} a} d y+\frac{1}{\varepsilon} \int_{x}^{1} b(y)\left[\int_{x}^{y} e^{(1 / \varepsilon) \int_{y}^{\xi} a} d \xi\right] V_{k}(y) d y \\
& -\frac{1}{\varepsilon} \int_{x}^{1}\left[\int_{x}^{y} e^{(1 / \varepsilon) \int_{y}^{\xi} a} d \xi\right] v_{k-1}^{\prime \prime}(y) d y .
\end{aligned}
$$

Evaluating at $x=0$ and using the other boundary condition $V_{k}(0)=0$, yields

$$
V_{k}^{\prime}(1)=\frac{\frac{1}{\varepsilon} \int_{0}^{1} b(y)\left[\int_{0}^{y} e^{(1 / \varepsilon) f_{y}^{\xi} a} d \xi\right] V_{k}(y) d y-\frac{1}{\varepsilon} \int_{0}^{1}\left[\int_{0}^{y} e^{(1 / \varepsilon) f_{y}^{\xi} a} d \xi\right] v_{k-1}^{\prime \prime}(y) d y}{\int_{0}^{1} e^{-(1 / \varepsilon) \int_{y}^{1} a} d y} .
$$

Now $\left\|V_{k}\right\|_{\infty}$ can be bounded independently of $\varepsilon$ for $\varepsilon$ sufficiently small [10], $v_{k-1}^{\prime \prime}$ is independent of $\varepsilon$ (and bounded if $a, b$, and $f$ are sufficiently smooth), and the denominator can be bracketed,

$$
\frac{\varepsilon}{\bar{a}}\left(1-e^{-(1 / \varepsilon) \int_{0}^{1} a}\right) \leqslant \int_{0}^{1} e^{-(1 / \varepsilon) \int_{y}^{1} a} d y \leqslant \frac{\varepsilon}{\underline{a}}\left(1-e^{-(1 / \varepsilon) \int_{0}^{1} a}\right),
$$

where $\underline{a} \equiv \min \{a(x): 0 \leqslant x \leqslant 1\}$ and $\bar{a} \equiv \max \{a(x): 0 \leqslant x \leqslant 1\}$. It follows that $V_{k}^{\prime}(1)=\mathcal{O}\left(\varepsilon^{-1}\right)$; therefore, from (2.3), $\left\|V_{k}^{\prime}\right\|_{\infty}=\mathcal{O}\left(\varepsilon^{-1}\right)$. Successive differentiations of (2.3) yield that $\left\|V_{k}^{(p)}\right\|_{\infty}=\mathcal{O}\left(\varepsilon^{-p}\right), p=2, \ldots, k$. We conclude that for $a, b$, and $f$ sufficiently smooth,

$$
\|v\|_{\infty},\left\|v^{\prime}\right\|_{\infty}, \ldots,\left\|v^{(k)}\right\|_{\infty} \leqslant C
$$

where $C$ is independent of $\varepsilon$.

The $w$-problem can be handled similarly. Two linearly independent solutions of the homogeneous equations $L w=0$ can be constructed in the form

$$
y(x ; \varepsilon)=y_{0}(x)+y_{1}(x) \varepsilon+\cdots+y_{k-1}(x) \varepsilon^{k-1}+Y_{k}(x ; \varepsilon) \varepsilon^{k}
$$

and

$$
z(x ; \varepsilon)=e^{-(1 / \varepsilon) \int_{x}^{1} a}\left(z_{0}(x)+z_{1}(x) \varepsilon+\cdots+z_{k-1}(x) \varepsilon^{k-1}+Z_{k}(x ; \varepsilon) \varepsilon^{k}\right),
$$

where the component functions here satisfy

$$
\begin{array}{rc}
a(x) y_{0}^{\prime}+b(x) y_{0}=0, \quad-\left(a(x) z_{0}\right)^{\prime}+b(x) z_{0}=0, \\
y_{0}(1)=1, & z_{0}(1)=1, \\
a(x) y_{j}^{\prime}+b(x) y_{j}=y_{j-1}^{\prime \prime}, & -\left(a(x) z_{j}\right)^{\prime}+b(x) z_{j}=z_{j-1}^{\prime \prime}, \\
y_{j}(1)=0, \quad j=1, \ldots, k-1, & z_{j}(1)=0, \quad j=1, \ldots, k-1,
\end{array}
$$


and

$$
\begin{gathered}
-\varepsilon Y_{k}^{\prime \prime}+a(x) Y_{k}^{\prime}+b(x) Y_{k}=y_{k-1}^{\prime \prime}, \quad-\varepsilon Z_{k}^{\prime \prime}-\left(a(x) Z_{k}\right)^{\prime}+b(x) Z_{k}=z_{k-1}^{\prime \prime}, \\
Y_{k}(0)=Y_{k}(1)=0, \quad Z_{k}(0)=Z_{k}(1)=0 .
\end{gathered}
$$

These two solutions can be combined to form a solution of the $w$-problem, and the remainder functions $Y_{k}$ and $Z_{k}$ can be analyzed exactly as before. The result follows.

The decomposition (2.1) is a standard sort of splitting for these problems, and the asymptotic correctness of the expansions has been rigorously established before (see, for example, [10], [27], or [32]). However, we could find no reference for the observation on the boundedness of the derivatives of the $A$ and $B$ functions, and we rely heavily on this in the development that follows. Also, we mention that (2.1) implies the inequalities

$$
\left|u^{(j)}(x)\right| \leqslant C\left(1+\frac{1}{\varepsilon^{j}} e^{-(1 / \varepsilon) \int_{x}^{1} a}\right), \quad j=0, \ldots, k,
$$

where $C$ is independent of $\varepsilon$. This type of a priori estimate is used often in the literature.

3. The Finite-Difference Scheme. We wish to construct compact finite-difference approximations to (1.1). For simplicity we use a uniform mesh $x_{i}=i h, i=0, \ldots, n$, $h=1 / n$. We construct a discretization of the form

$$
\begin{gathered}
L_{h} u_{i}^{h} \equiv \alpha_{i,-1} u_{i-1}^{h}+\alpha_{i, 0} u_{i}^{h}+\alpha_{i, 1} u_{i+1}^{h}=\sum_{j=1}^{J} \beta_{i, j} f\left(\xi_{i, j}\right), \quad i=1, \ldots, n-1, \\
u_{0}^{h}=g_{0}, \quad u_{n}^{h}=g_{1} .
\end{gathered}
$$

The points $\xi_{i, 1}, \ldots, \xi_{i, J}$ are auxiliary evaluation points (also called HODIE points [21]). They are distinct and lie between $x_{i-1}$ and $x_{i+1}$; some of them may coincide with mesh points.

The coefficients, $\alpha_{i,-1}, \alpha_{i, 0}$ and $\alpha_{i, 1}$, and weights, $\beta_{i, 1}, \ldots, \beta_{i, J}$, are determined so that the scheme is locally exact on some $(J+2)$-dimensional space of approximating functions, in the sense that this space is contained in the kernel of the local truncation operator

$$
T_{i}[\phi] \equiv L_{h} \phi_{i}-\sum_{j=1}^{J} \beta_{i, j} L \phi\left(\xi_{i, j}\right),
$$

subject to the normalization

$$
\sum_{j=1}^{J} \beta_{i, j}=1
$$

This leads to a local linear algebraic system to determine the $\alpha$ 's and $\beta$ 's. For $J=1$ and $\xi_{i, 1}=x_{i}$, the scheme that is exact on $\left\{1, x, x^{2}\right\}$ is precisely the standard central-difference approximation, while for $J=1$ and $\xi_{i, 1}=x_{i}$, one gets the AllenSouthwell scheme [3] by requiring exactness on $\left\{1, x, \exp \left(a\left(x_{i}\right) x / \varepsilon\right)\right\}$.

This general approach to finite differences has been analyzed by Lynch and Rice [21], who refer to it as the HODIE (High-Order Differences via Identity Expansions) Method, and Doedel [11] (see also [7], where a variation of this is referred to as an 
Operator Compact Implicit Scheme). These authors have shown that for a problem such as (1.1), with $\varepsilon$ fixed, if one uses $J$ evaluation points and imposes exactness on $\left\{1, x, \ldots, x^{J+1}\right\}$, then for $h$ sufficiently small, the local linear system that defines the coefficients and weights is nonsingular and produces a stable approximation with discretization error at least $\mathcal{O}\left(h^{J}\right)$.

The difficulty with the approach as it applies to our problem (1.1) is that no matter how large $J$ is taken above, the resulting discretization is not even uniformly $\mathcal{O}(h)$. That is, while $\left\|e^{h}\right\| \leqslant c h^{J}, c$ depends on $\varepsilon$ and blows up as $\varepsilon \rightarrow 0$ and $\sup \left(h^{-1}\left\|e^{h}\right\|\right) \rightarrow \infty$ as $\varepsilon \rightarrow 0$. To remedy this situation here we emulate (2.1) and construct our scheme to be exact on

$$
\left\{1, x, \ldots, x^{p}, \exp \left(\frac{1}{\varepsilon} \int^{x} a\right), x \exp \left(\frac{1}{\varepsilon} \int^{x} a\right), \ldots, x^{p-1} \exp \left(\frac{1}{\varepsilon} \int^{x} a\right)\right\} .
$$

It is natural to refer to such a scheme as an exponentially fitted HODIE scheme. To produce a well-posed local linear system with such a family of approximating functions, it is necessary that the auxiliary points be distributed with $p$ in the upwind subinterval $\left[x_{i-1}, x_{i}\right]$ and $(p-1)$ downwind in $\left(x_{i}, x_{i+1}\right]$. We will analyze here the special (natural) case of equally spaced points,

$$
\begin{gathered}
\xi_{i, 1}=x_{i}, \quad p=1, \\
\xi_{i, j}=x_{i-1}+\frac{j-1}{p-1} h, \quad j=1, \ldots, 2 p-1, p=2,3, \ldots
\end{gathered}
$$

We now prove that for these points the local system is nonsingular for all $h$ sufficiently small, independent of $\varepsilon$.

THEOREM 3.1. Let the positive integer $p$ be given. If the coefficient functions $a$ and $b$ in (1.1) are sufficiently smooth (in addition to satisfying $a(x) \geqslant \underline{a}>0$ ), then for all $h$ sufficiently small, independent of $\varepsilon$ and $i$, the coefficients $\alpha_{i,-1}, \alpha_{i, 0}$, and $\alpha_{i, 1}$ and weights $\beta_{i, 1}, \ldots, \beta_{i, 2 p-1}$ in the finite-difference approximation (3.1) are uniquely determined by the conditions of exactness on the family of functions $\left\{1, x, \ldots, x^{p}\right.$, $\left.\exp \left(\frac{1}{\varepsilon} \int^{x} a\right), x \exp \left(\frac{1}{\varepsilon} \int^{x} a\right), \ldots, x^{p-1} \exp \left(\frac{1}{\varepsilon} \int^{x} a\right)\right\}$, subject to the normalization (3.2) and distribution requirement (3.3), and satisfy

(a) $\left|\alpha_{i,-1}+\alpha_{i, 0}+\alpha_{i, 1}\right| \leqslant M<\infty$,

(b) $h\left(\alpha_{i, 1}-\alpha_{i,-1}\right) \geqslant m>0$,

(c) $\alpha_{i,-1} \leqslant-\varepsilon / h^{2} \leqslant \alpha_{i, 1} \leqslant 0$,

where $m$ and $M$ are constants that do not depend on $h, \varepsilon$, or $i$.

The proof of this theorem is lengthy and detailed and is relegated to the Appendix in the Supplements section of this issue. Briefly, it proceeds by carefully analyzing the concerned linear system for $h$ small in the three cases $h / \varepsilon \rightarrow 0,0<\rho \leqslant h / \varepsilon \leqslant \bar{\rho}$ $<\infty$, and $h / \varepsilon \rightarrow \infty$. It is a consequence of this analysis that $\beta_{i, p+1}, \ldots, \beta_{i, 2 p-1}$, and $\alpha_{i, 1}$ all go to zero as $h / \varepsilon \rightarrow \infty$, and the finite-difference scheme converges to a 2-point $\mathcal{O}\left(h^{p}\right)$ discretization of the form

$$
\alpha_{i,-1} u_{i-1}^{h}+\alpha_{i, 0} u_{i}^{h}=\sum_{j=1}^{p} \beta_{i, j} f\left(\xi_{i, j}\right)
$$


for the reduced equation

$$
a(x) u^{\prime}+b(x) u=f(x)
$$

that is exact on $\left(1, x, \ldots, x^{p}\right)$ : The estimates $(3.4 \mathrm{a}, \mathrm{b}$, and $\mathrm{c})$ are required in the stability analysis in Section 5.

4. Truncation Error Analysis. We now analyze the truncation error $\tau$ defined by

$$
\begin{aligned}
\tau_{i} \equiv T_{i}[u]= & \alpha_{i,-1} u\left(x_{i-1}\right)+\alpha_{i, 0} u\left(x_{i}\right)+\alpha_{i, 1} u\left(x_{i+1}\right) \\
& -\sum_{j=1}^{2 p-1} \beta_{i, j} L u\left(\xi_{i, j}\right), \quad i=1, \ldots, n-1 .
\end{aligned}
$$

Here $u$ is the true solution of (1.1), and $\alpha_{i,-1}, \ldots, \beta_{i, 2 p-1}$ are the coefficients and weights of an exponentially fitted HODIE scheme constructed as in Section 3. This, when combined with an appropriate stability result, can be used to appraise the discretization error $e^{h}$, where $e_{i}^{h} \equiv u\left(x_{i}\right)-u_{i}^{h}$, since $L_{h} e_{i}^{h}=\tau_{i}$. Our main result on the truncation error is the following.

Theorem 4.1. Let the positive integer $p$ be given, and let $\tau$ denote the local truncation error (4.1) in an exponentially fitted HODIE approximation to (1.1) of the form (3.1) constructed to be exact on

$$
\left\{1, x, \ldots, x^{p}, \exp \left(\frac{1}{\varepsilon} \int^{x} a\right), x \exp \left(\frac{1}{\varepsilon} \int^{x} a\right), \ldots, x^{p-1} \exp \left(\frac{1}{\varepsilon} \int^{x} a\right)\right\}
$$

and subject to the normalization (3.2) and distribution requirement (3.3). If the functions $a, b$, and $f$ in (1.1) are sufficiently smooth, then there exists a constant $c$ that is independent of $\varepsilon, h$, and $i$, such that for all $\varepsilon$ sufficiently small and all $h$ sufficiently small (independent of $\varepsilon$ ),

$$
\left|\tau_{i}\right| \leqslant \operatorname{ch}^{p}\left(1+\frac{1}{\varepsilon} \exp \left(-\frac{1}{\varepsilon} \int_{x_{i}}^{1} a\right)\right), \quad i=1, \ldots, n-1 .
$$

Proof. As in the proof of Theorem 3.1, we consider the interval $[-h, h]$ (suppress the subscript $i)$ and denote $E(x)=\exp \left(\frac{1}{\varepsilon} \int_{0}^{x} a\right)$. It is a rather straightforward consequence of the leading order behavior of the finite-difference coefficients $\alpha_{-1}$ and $\alpha_{1}$ (as analyzed in the proof of Theorem 3.1) that they satisfy

$$
\left|\alpha_{-1}\right|,\left|\alpha_{1}\right|, E(-h)\left|\alpha_{-1}\right|, E(h)\left|\alpha_{1}\right| \leqslant C_{1} \begin{cases}\frac{\varepsilon}{h^{2}}, & h \leqslant \varepsilon, \\ \frac{1}{h}, & \varepsilon \leqslant h,\end{cases}
$$

for some absolute constant $C_{1}$. Likewise, the weights $\beta_{1}, \ldots, \beta_{2 p-1}$ satisfy

$$
\left|\beta_{1}\right|, \ldots,\left|\beta_{p}\right|, E\left(\xi_{p+1}\right)\left|\beta_{p+1}\right|, \ldots, E\left(\xi_{2 p-1}\right)\left|\beta_{2 p-1}\right| \leqslant C_{2} .
$$

Now we know from Theorem 2.2 that for $\varepsilon$ sufficiently small, $u$ admits the representation

$$
u(x)=A(x)+B(x) \exp \left(-\frac{1}{\varepsilon} \int_{x}^{1} a\right)
$$

where $A$ and $B$ are smooth functions (if $a, b$, and $f$ are sufficiently smooth) that can be bounded together with their derivatives up to any given finite order, independently of $\varepsilon$. We can estimate the leading order terms of the truncation error 
as follows. For $h \geqslant \varepsilon$ we have (using the above estimates on the $\alpha$ 's and $\beta$ 's)

$$
\begin{aligned}
\left|T\left[x^{p+1}\right]\right|= & \mid \alpha_{-1}(-1)^{p+1} h^{p+1}+\alpha_{1} h^{p+1} \\
& -\sum \beta_{j}\left[-\varepsilon(p+1) p \xi_{j}^{p-1}+a\left(\xi_{j}\right)(p+1) \xi_{j}^{p}+b\left(\xi_{j}\right) \xi_{j}^{p+1}\right] \mid \\
\leqslant & c_{3} h^{p}
\end{aligned}
$$

and

$$
\begin{aligned}
\left|T\left[x^{p} E(x)\right]\right|= & \mid \alpha_{-1}(-1)^{p} h^{p} E(-h)+\alpha_{1} h^{p} E(h) \\
& -\sum \beta_{j} E\left(\xi_{j}\right)\left[-\varepsilon \rho(p-1) \xi_{j}^{p-2}-a\left(\xi_{j}\right) p \xi_{j}^{p-1}+\left(b-a^{\prime}\right)\left(\xi_{j}\right) \xi_{j}^{p}\right] \mid \\
\leqslant & c_{4} h^{p-1} \leqslant c_{4} \frac{h^{p}}{\varepsilon} .
\end{aligned}
$$

To handle the case $h \leqslant \varepsilon$, we expand

$$
E(x)=\exp \left(\frac{1}{\varepsilon} \int_{0}^{x} a\right)=1+d_{1} x+d_{2} x^{2}+\cdots,
$$

where $d_{k}=\mathcal{O}\left(\varepsilon^{-k}\right), k=1,2 \ldots$ Then $x^{p+1}$ can be written

$$
x^{p+1}=\frac{1}{d_{p+1}}\left\{E(x)-\left[1+d_{1} x+\cdots+d_{p} x^{p}+d_{p+2} x^{p+2}+\cdots\right]\right\} .
$$

And it follows that

$$
\begin{aligned}
\left|T\left[x^{p+1}\right]\right| & \leqslant c_{5}\left|\frac{d_{p+2}}{d_{p+1}}\right|\left|T\left[x^{p+2}\right]\right| \\
& \leqslant c_{6} \frac{1}{\varepsilon}\left(\frac{\varepsilon}{h^{2}} \cdot h^{p+2}+\varepsilon h^{p}+h^{p+2}\right) \leqslant c_{7} h^{p} .
\end{aligned}
$$

Finally, still for $h \leqslant \varepsilon$, we have

$$
\begin{aligned}
\left|T\left[x^{p} E(x)\right]\right| & =\left|T\left[x^{p}\left(1+d_{1} x+\cdots\right)\right]\right| \\
& \leqslant c_{8}\left|d_{1}\right|\left|T\left[x^{p+1}\right]\right| \leqslant c_{9} \frac{1}{\varepsilon} h^{p} .
\end{aligned}
$$

The truncation error can be bounded via these leading-order terms. Combining these various estimates, we get

$$
\begin{aligned}
\left|\tau_{i}\right| & =\left|T_{i}[u]\right|=\left|T_{i}[A]+T_{i}\left[B \exp \left(-\frac{1}{\varepsilon} \int_{x}^{1} a\right)\right]\right| \\
& \leqslant c_{10}\left\{\left|T_{i}\left[\left(x-x_{i}\right)^{p+1}\right]\right|+\left|T_{i}\left[\left(x-x_{i}\right)^{p} \exp \left(-\frac{1}{\varepsilon} \int_{x}^{1} a\right)\right]\right|\right\} \\
& \leqslant c_{11}\left\{h^{p}+\exp \left(-\frac{1}{\varepsilon} \int_{x_{i}}^{1} a\right)\left|T_{i}\left[\left(x-x_{i}\right)^{p} \exp \left(\frac{1}{\varepsilon} \int_{x_{i}}^{x} a\right)\right]\right|\right\} \\
& \leqslant c^{p}\left(1+\frac{1}{\varepsilon} \exp \left(-\frac{1}{\varepsilon} \int_{x_{i}}^{1} a\right)\right) .
\end{aligned}
$$

We mention that the reason we are able to get by with one less local exponential function (and essentially trade a power of $h$ for a factor of $1 / \varepsilon$ ) is that the function in parentheses in our bound for $\left|\tau_{i}\right|$ has a uniformly bounded $L^{1}$-norm; our stability 
estimate involves a discrete 1-norm of the truncation error, and we get the desired bound on the error $e^{h}$. We take up this aspect now.

5. Discretization Error Analysis. We can use the general stability theorems in [25] to analyze our exponentially fitted HODIE schemes. We first paraphrase an appropriate version of those results as they apply to our particular problem.

THEOREM 5.1. Let a uniform mesh $x_{i}=i h, i=0, \ldots, n, h=1 / n$ on $[0,1]$ be given. Let $L_{h}$ be a finite-difference operator of the form

$$
L_{h} v_{i}^{h} \equiv \alpha_{i,-1} v_{i-1}^{h}+\alpha_{i, 0} v_{i}^{h}+\alpha_{i, 1} v_{i+1}^{h},
$$

where the coefficients $\alpha_{i,-1}, \alpha_{i, 0}$, and $\alpha_{i, 1}$ satisfy, for some positive constants $m$ and $M$,

(a) $h\left(\alpha_{i, 1}-\alpha_{i,-1}\right) \geqslant m$,

(b) $\left|\alpha_{i,-1}+\alpha_{i, 0}+\alpha_{i, 1}\right| \leqslant M$,

(c) $\alpha_{i,-1} \leqslant-\varepsilon / h^{2} \leqslant \alpha_{i, 1} \leqslant 0$.

Let $L$ denote the singularly perturbed differential operator of (1.1), and let $y_{0}$ and $y_{1}$ denote solutions of the associated homogeneous problems

$$
\begin{array}{cc}
L y_{0}=L y_{1}=0, & 0<x<1, \\
y_{0}(0)=1, & y_{1}(0)=0, \\
y_{0}(1)=0, & y_{1}(1)=1 .
\end{array}
$$

Let $\|\cdot\|_{h, \infty}$ and $\|\cdot\|_{h, 1}$ denote the discrete $\infty$-norm and 1-norm defined by

$$
\left\|v^{h}\right\|_{h, \infty} \equiv \max \left\{\left|v_{i}^{h}\right|: i=0, \ldots, n\right\}
$$

and

$$
\left\|v^{h}\right\|_{h, 1} \equiv h \sum_{i=1}^{n-1}\left|v_{i}^{h}\right| .
$$

Let the original differential operator $L$ satisfy, for all $0<\varepsilon \leqslant \varepsilon_{0}$ and for all $v$ in $C^{2}[0,1]$,

$$
\|v\|_{\infty} \leqslant c\left\{\|L v\|_{1}+|v(0)|+|v(0)|\right\},
$$

where $c$ is a constant that does not depend on $\varepsilon$.

It then follows that there is an absolute tolerance $\eta>0$, which does not depend on $\varepsilon$ or $h$, such that, if the combined residual (consistency error) below satisfies

$$
\left\|\left(L_{h}-L\right) y_{0}\right\|_{h, 1}+\left\|\left(L_{h}-L\right) y_{1}\right\|_{h, 1} \leqslant \eta, \quad 0<\varepsilon \leqslant \varepsilon_{0},
$$

then for all mesh functions $v^{h}$ and $0<\varepsilon \leqslant \varepsilon_{0}$, we have

$$
\left\|v^{h}\right\|_{h, \infty} \leqslant c^{\prime}\left\{\left\|L_{h} v^{h}\right\|_{h, 1}+\left|v_{0}^{h}\right|+\left|v_{n}^{h}\right|\right\}
$$

where $c^{\prime}$ is a constant that does not depend on $\varepsilon$.

This is merely a restatement of Theorem 12 of [25]. In our case, the operators are second order, the mesh uniform, and the norms, \|\|$\cdot\|\|_{\varepsilon}$ and $\|\mid \cdot\| \|_{H, \varepsilon}$ in [25], are taken to be continuous and discrete $\infty$-norms. Also, our formulation differs from that in [25] in the sign conditions on $\varepsilon$ and $a: L_{\varepsilon} u \equiv \varepsilon u^{\prime \prime}+a(x) u^{\prime}+b(x) u$ in [25] versus $L u \equiv-\varepsilon u^{\prime \prime}+a(x) u^{\prime}+b(x) u$ here, in (1.1). This changes, under the transformation $x \mapsto 1-x$, the forward differences in [25, Theorem 2] to backward differences; the inequalities (a), (b), and (c) above are equivalent to the conditions (i), (ii), and (iii) of that theorem. 
We are now in a position to uniformly bound the discretization error in our exponentially fitted HODIE approximations.

THEOREM 5.2. Let the positive integer $p$ be given. Let $e^{h}$ denote the discretization error $e_{i}^{h} \equiv u\left(x_{i}\right)-u_{i}^{h}$, where $u$ is the true solution of (1.1) and $u^{h}$ is the finite-difference approximation to $u$ that solves the exponentially fitted HODIE scheme (3.1), with $J=2 p-1$, constructed as in Section 3. If the coefficient functions $a, b$, and $f$ in (1.1) are sufficiently smooth, then there exists a constant $c$ that is independent of $\varepsilon$ and $h$ such that for all $\varepsilon$ sufficiently small and all $h$ sufficientily small (independent of $\varepsilon$ )

$$
\left\|e^{h}\right\|_{h, x} \leqslant c h^{p} \text {. }
$$

Proof. Let $\tau$ denote the truncation error (4.1); then $e^{h}$ satisfies

$$
L_{h} e_{i}^{h}=\tau_{i}, \quad i=1 \ldots, n-1, \quad e_{0}^{h}=e_{n}^{h}=0 .
$$

Theorem 4.1 then implies that for $\varepsilon$ and $h$ sufficiently small,

$$
\left|L_{h} e_{i}^{h}\right| \leqslant c_{1} h^{p}\left(1+\frac{1}{\varepsilon} \exp \left(-\frac{1}{\varepsilon} \int_{x_{i}}^{1} a\right)\right) .
$$

Observe that

$$
\begin{aligned}
\left\|\exp \left(-\frac{1}{\varepsilon} \int_{x_{i}}^{1} a\right)\right\|_{h, 1} & =h \sum_{i=1}^{n-1}\left|\exp \left(-\frac{1}{\varepsilon} \int_{x_{i}}^{1} a\right)\right|<\int_{0}^{1} \exp \left(-\frac{1}{\varepsilon} \int_{x}^{1} a\right) d x \\
& \leqslant \frac{\varepsilon}{\underline{a}}\left(1-\exp \left(-\frac{1}{\varepsilon} \int_{0}^{1} a\right)\right) .
\end{aligned}
$$

Thus we have $\left\|L_{h} e^{h}\right\|_{h, 1} \leqslant c_{2} h^{p}$.

We know (Theorem 2.1) that the original problem satisfies the right kind of stability estimate, for $\varepsilon$ sufficiently small. And we know (Theorem 3.1) that our finite-difference scheme is well defined and satisfies conditions (a), (b), (c) of Theorem 5.1, for $\varepsilon$ and $h$ sufficiently small. Our result will follow from Theorem 5.1; all we require is that the consistency error in (5.2) be sufficiently small. But this expression is nothing more than the sum of the mesh 1-norms of the truncation errors in the approximations of problems of the type (1.1) with $f(x) \equiv 0$ and $g_{0}=1, g_{1}=0$ (in the case of $y_{0}$ ) and $g_{0}=0, g_{1}=1$ (in the case of $y_{1}$ ). Theorem 4.1 then implies that the consistency error is at least $\mathcal{O}(h)$. So the needed inequality (5.2) will be satisfied for $h$ sufficiently small, and the theorem is proved.

The construction and analysis of these schemes thus far has assumed explicit knowledge of the derivative and definite integrals of the coefficient function $a$ in (1.1). We now show that if we approximate this needed information in a natural, numerical way, then we get the same uniform order of convergence. Suppose that in the subinterval $\left[x_{i}, x_{i+1}\right]$, we approximate $a$ locally by the polynomial $q_{i}$ of degree $p-1$ that interpolates to $a$ at the auxiliary evaluation points used in that subinterval, viz., $\xi_{i, p}=\xi_{i+1,1}=x_{i}, \xi_{i, p+1}=\xi_{i+1,2}, \ldots, \xi_{i, 2 p-1}=\xi_{i+1, p}=x_{i+1}$. We accept the integral and derivatives of $q_{i}$ as approximations to those of $a$ and get

$$
\begin{aligned}
& \int_{x_{i}}^{\xi_{i, j}} a=\int_{x_{i}}^{\xi_{i, j}} q_{i}+\mathcal{O}\left(h^{p+1}\right), \quad a^{\prime}\left(\xi_{i, j}\right)=q_{i}^{\prime}\left(\xi_{i, j}\right)+\mathcal{O}\left(h^{p-1}\right), \\
& j=p, \ldots, 2 p-1,
\end{aligned}
$$


and

$$
\begin{array}{r}
\int_{x_{i+1}}^{\xi_{i+1, j}} a=\int_{x_{i+1}}^{\xi_{i+1, j}} q_{i}+\mathcal{O}\left(h^{p+1}\right), \quad a^{\prime}\left(\xi_{i+1, j}\right)=q_{i}^{\prime}\left(\xi_{i+1, j}\right)+\mathcal{O}\left(h^{p-1}\right), \\
j=1, \ldots, p .
\end{array}
$$

We then have the following theorem.

THEOREM 5.3. Let the positive integer $p$ be given. Let an approximate exponentially fitted HODIE discretization of (1.1) be constructed as in Section 3 but with the needed integrals and derivatives of a replaced by the numerical approximations indicated above. If $a, b$, and $f$ are sufficiently smooth, then for all $h$ sufficiently small, independent of $\varepsilon$, these finite-difference schemes are well defined and stable, and the associated discretization error is uniformly $\mathcal{O}\left(h^{p}\right)$.

Proof. All that we need to do here is to observe that our approximate scheme is a regular exponentially fitted HODIE scheme for the approximate problem

$$
\begin{gathered}
\tilde{L} \tilde{u} \equiv-\varepsilon \tilde{u}^{\prime \prime}+\tilde{a}(x) \tilde{u}^{\prime}+b(x) \tilde{u}=f(x), \quad 0<x<1, \\
\tilde{u}(0)=g_{0}, \quad \tilde{u}(1)=g_{1},
\end{gathered}
$$

where $\tilde{a}$ is a continuous piecewise-polynomial approximation to $a$ given by $a(x)=$ $q_{i}(x), x_{i} \leqslant x \leqslant x_{i+1}$ (here $q_{i}$ is the local polynomial interpolant introduced above).

Now by construction, $\|a-\tilde{a}\|_{\infty}=\mathcal{O}\left(h^{p}\right)$ (for $a$ sufficiently smooth), and it follows that $\tilde{a}(x) \geqslant \underline{a} / 2>0$, say, for all $h$ sufficiently small. Thus our previous analysis implies that the scheme is well defined and stable; we need only show that $\|u-\tilde{u}\|_{\infty}$ is uniformly $\mathcal{O}\left(h^{p}\right)$. But this follows since $\tilde{e} \equiv u-\tilde{u}$ satisfies

$$
\begin{gathered}
\tilde{L} \tilde{e}=(a(x)-\tilde{a}(x)) u^{\prime}(x), \quad 0<x<1, \\
\tilde{e}(0)=\tilde{e}(1)=0,
\end{gathered}
$$

from which we obtain

$$
\|\tilde{e}\|_{\infty} \leqslant c\|a-\tilde{a}\|_{\infty}\left\|u^{\prime}\right\|_{1} \leqslant c^{\prime} h^{p}
$$

Here we have used the facts that the operators $\tilde{L}$ satisfy a uniform stability estimate of the type in Theorem 2.1 (for all $h$ sufficiently small), $u^{\prime}$ satisfies (from Theorem 2.2)

$$
\left|u^{\prime}(x)\right| \leqslant c\left(1+\frac{1}{\varepsilon} \exp \left(-\frac{1}{\varepsilon} \int_{x}^{1} a\right)\right),
$$

and $\left\|\varepsilon^{-1} \exp \left(-\frac{1}{\varepsilon} \int_{x}^{1} a\right)\right\|_{1} \leqslant c$, uniformly in $\varepsilon$.

We see that the same uniform order of convergence can be obtained using only those point evaluations of $a$ (and $b$ and $f$ ) that were already required in the original formulation; $p$-values per mesh subinterval yield a uniform $\mathcal{O}\left(h^{p}\right)$-scheme.

6. Numerical Results and Remarks. In this section are reported a small sample of numerical experiments to demonstrate the behavior of these exponentially fitted HODIE schemes. The results below are for the following model problem,

$$
\begin{gathered}
-\varepsilon u^{\prime \prime}+\frac{1}{x+1} u^{\prime}+\frac{1}{x+2} u=f(x), \quad 0<x<1, \\
u(0)=1+2^{-1 / \varepsilon}, \quad u(1)=e+2,
\end{gathered}
$$


with $f$ corresponding to the true solution

$$
u(x)=e^{x}+2^{-1 / \varepsilon}(x+1)^{1+1 / \varepsilon} .
$$

Approximate solutions were computed on a CDC 6600 in single precision (around 14 decimal digit accuracy) using schemes constructed as in Section 3.

Discretization errors were tabulated using a uniform mesh spacing $h=1 / n$, $n=4,8,16, \ldots, 1024$ while holding the ratio $\rho \equiv h / \varepsilon$ fixed, that is, $\varepsilon \rightarrow 0$ as $h \rightarrow 0$ with $h / \varepsilon \equiv$ constant. The experiments were constructed in this way to make it easier to observe the uniform convergence rates, which were approximated using

$$
p \approx \tilde{p} \equiv \log _{2} \frac{\left\|e_{n}\right\|}{\left\|e_{2 n}\right\|}, \quad n=4,8, \ldots, 512 .
$$

As an illustration, consider the uniform $\mathcal{O}(h)$-scheme. These results are contained in Table 1 below.

This table exhibits one of the standard features of these schemes, namely, until $\varepsilon$ gets small enough for the problem to actually appear to be singularly perturbed, here around $\varepsilon<\frac{1}{4}\left(\rho=2^{-4}\right.$ and $\left.h=1 / 128, \ldots\right)$, one observes the nonuniform (fixed $\varepsilon$ ) convergence rate, here $\mathcal{O}\left(h^{2}\right)$. Once this threshold is passed, however, the $\mathcal{O}(h)$ convergence is clearly indicated. Also, for each $n$, the maximum $\|e\|_{\infty}$ occurs for $2^{-2} \leqslant \rho \leqslant 2^{2}$, with the errors decreasing outside of this range. This leads us to define (and approximate)

$$
\|e\|_{\infty} \equiv \max _{0<\rho<\infty}\|e\|_{\infty} \approx \max _{\rho=2^{-4}, \ldots, 2^{6}}\|e\|_{\infty} .
$$

TABLE 1

Maximum errors and approximate uniform convergence rates for the $\mathcal{O}(h)$ exponentially fitted HODIE scheme applied to (6.1)

\begin{tabular}{r|c|c|c|c|c|c}
\hline \multirow{2}{*}{$n$} & \multicolumn{2}{|c|}{$\rho=2^{-4}$} & \multicolumn{2}{c|}{$\rho=2^{-2}$} & \multicolumn{2}{c}{$\rho=2^{0}$} \\
\cline { 2 - 7 } & $\|e\|_{\infty}$ & $\tilde{p}$ & $\|e\|_{\infty}$ & $\tilde{p}$ & $\|e\|_{\infty}$ & $\tilde{p}$ \\
\hline 4 & $.50(-1)$ & .97 & $.44(-1)$ & 1.13 & $.22(-1)$ & 1.60 \\
8 & $.25(-1)$ & 1.06 & $.20(-1)$ & 1.58 & $.73(-2)$ & -.86 \\
16 & $.12(-1)$ & 1.28 & $.66(-2)$ & 1.67 & $.13(-1)$ & .29 \\
32 & $.53(-2)$ & 1.63 & $.21(-2)$ & -.82 & $.11(-1)$ & .64 \\
64 & $.17(-2)$ & 1.69 & $.37(-2)$ & .30 & $.69(-2)$ & .81 \\
128 & $.53(-3)$ & -.81 & $.30(-2)$ & .65 & $.40(-2)$ & .89 \\
256 & $.93(-3)$ & .30 & $.19(-2)$ & .81 & $.21(-2)$ & .94 \\
512 & $.76(-3)$ & .65 & $.11(-2)$ & .89 & $.11(-2)$ & .97 \\
1024 & $.48(-3)$ & & $.58(-3)$ & & $.57(-3)$ & \\
\hline
\end{tabular}

\begin{tabular}{r|l|l|l|l|l|l}
\hline \multirow{2}{*}{$n$} & \multicolumn{2}{|c|}{$\rho=2^{2}$} & \multicolumn{2}{c|}{$\rho=2^{4}$} & \multicolumn{2}{c}{$\rho=2^{6}$} \\
\cline { 2 - 7 } & $\|e\|_{\infty}$ & $\tilde{p}$ & \multicolumn{1}{c|}{$\|e\|_{\infty}$} & $\tilde{p}$ & $\|e\|_{\infty}$ & $\tilde{p}$ \\
\hline 4 & $.34(-1)$ & .18 & $.27(-1)$ & .82 & $.12(-1)$ & 1.13 \\
8 & $.30(-1)$ & .64 & $.15(-1)$ & .93 & $.54(-2)$ & 1.14 \\
16 & $.19(-1)$ & .81 & $.81(-2)$ & .97 & $.25(-2)$ & 1.09 \\
32 & $.11(-1)$ & .88 & $.42(-2)$ & .98 & $.12(-2)$ & 1.05 \\
64 & $.59(-2)$ & .94 & $.21(-2)$ & .99 & $.56(-3)$ & 1.03 \\
128 & $.31(-2)$ & .96 & $.11(-2)$ & 1.00 & $.27(-3)$ & 1.02 \\
256 & $.16(-2)$ & .98 & $.53(-3)$ & 1.00 & $.13(-3)$ & 1.01 \\
512 & $.80(-3)$ & .99 & $.27(-3)$ & 1.00 & $.67(-4)$ & 1.00 \\
1024 & $.40(-3)$ & & $.13(-3)$ & & $.33(-4)$ & \\
\hline
\end{tabular}


This "maximum-maximum" measurement of the error is tabulated below (Table 2) for the same model problem (6.1), using our uniform $\mathcal{O}\left(h^{p}\right)$-schemes with $p=1,2$, 3 , and 4 . These results clearly demonstrate the convergence rates predicted by the theory.

By way of concluding remarks we mention that investigations are underway generalizing this approach to other singular perturbation problems, including linear turning-point problems and nonlinear problems in one dimension and linear convection-diffusion problems in two dimensions. The applicability of the basic approach is quite general; essentially, if one has decent a priori information about the local behavior of the true solution of the problem, then this approach gives a way of incorporating that into the discretization.

TABLE 2

Maximum (over $\rho$ ) maximum errors and approximate uniform convergence rates for exponentially fitted HODIE schemes

$\mathcal{O}\left(h^{p}\right), p=1,2,3$, and 4 appied to $(6.1)$

\begin{tabular}{r|l|l|l|l|l|l|l|l}
\hline \multirow{2}{*}{$n$} & \multicolumn{2}{|c|}{$p=1$} & \multicolumn{2}{c|}{$p=2$} & \multicolumn{2}{c|}{$p=3$} & \multicolumn{2}{c}{$p=4$} \\
\cline { 2 - 8 } & $\|e\|_{\infty}$ & $\tilde{p}$ & $\|e\|_{\infty}$ & $\tilde{p}$ & $\|e\|_{\infty}$ & $\tilde{p}$ & $\|e\|_{\infty}$ & $\tilde{p}$ \\
\hline 4 & $.50(-1)$ & .73 & $.20(-2)$ & 1.72 & $.28(-4)$ & 2.68 & $.86(-6)$ & 3.73 \\
8 & $.30(-1)$ & .64 & $.60(-3)$ & 1.87 & $.43(-5)$ & 2.85 & $.65(-7)$ & 3.88 \\
16 & $.19(-1)$ & .81 & $.16(-3)$ & 1.94 & $.60(-6)$ & 2.93 & $.44(-8)$ & 3.94 \\
32 & $.11(-1)$ & .64 & $.43(-4)$ & 1.97 & $.79(-7)$ & 2.96 & $.29(-9)$ & 3.95 \\
64 & $.69(-2)$ & .81 & $.11(-4)$ & 1.98 & $.10(-7)$ & 2.98 & $.19(-10)$ & $*$ \\
128 & $.40(-2)$ & .89 & $.28(-5)$ & 1.99 & $.13(-8)$ & 2.98 & $*$ & $*$ \\
256 & $.21(-2)$ & .94 & $.70(-6)$ & 2.00 & $.16(-9)$ & 2.92 & $*$ & $*$ \\
512 & $.11(-2)$ & .89 & $.17(-6)$ & 2.00 & $.21(-10)$ & $*$ & $*$ & $*$ \\
1024 & $.58(-3)$ & - & $.44(-7)$ & - & $*$ & - & $*$ & - \\
\hline
\end{tabular}

The technical details of rigorous convergence proofs can be extremely difficult to overcome, even for the one-dimensional problems. Here we were able to get enough information out of an asymptotic representation of the solution (essentially the multiple scales expansion) to do our truncation error analysis. Also, each of these problems requires a new stability analysis, as the standard results for ordinary differential equations and elliptic partial differential equations do not apply uniformly in $\varepsilon$.

Department of Mathematics Southern Methodist University Dallas, Texas 75275

1. L. R. Abrahamsson, H. B. Keller \& H. O. Kreiss, "Difference approximations for singular perturbations of systems of ordinary differential equations," Numer. Math., v. 22, 1974, pp. 367-391.

2. L. AbrahamsSON \& S. Osher, "Monotone difference schemes for singular perturbation problems," SIAM J. Numer. Anal., v. 19, 1982, pp. 979-992.

3. D. N. DE G. Allen \& R. V. Southwell, "Relaxation methods applied to determine the motion in two dimensions of a viscous fluid past a fixed cylinder," Quart. J. Mech. Appl. Math., v. 8, 1955, pp. 129-145.

4. U. ASCHER \& R. WEISS, "Collocation for singular perturbation problems I: First order systems with constant coefficients," SIAM J. Numer. Anal., v. 20, 1983, pp. 537-557.

5. U. ASChER \& R. WeISS, "Collocation for singular perturbation problems. II: Linear first order systems without turning points,” Math. Comp., v. 43, 1984, pp. 157-187. 
6. A. O. H. AxelsSon, "Stability and error estimates of Galerkin finite-element approximations for convection-diffusion equations," IMA J. Numer. Anal., v. 1, 1981, pp. 329-345.

7. A. E. Berger, J. M. Solomon, M. Ciment, S. H. Leventhal \& B. C. Weinberg, "Generalized operator compact implicit schemes for boundary layer problems," Math. Comp., v. 35, 1980, pp. 695-731.

8. A. E. Berger, J. M. Solomon \& M. Ciment, "An analysis of a uniformly accurate difference method for a singular perturbation problem," Math. Comp., v. 37. 1981, pp. 79-94.

9. R. C. Y. ChIN \& R. KRASNY, "A hybrid asymptotic/finite-element method for stiff two-point boundary value problems," SIAM J. Sci. Statist. Comput., v. 4, 1983, pp. 229-243.

10. J. A. Cochran, "On the uniqueness of solutions of linear differential equations," J. Math. Anal. Appl., v. 22, 1968, pp. 418-426.

11. E. J. DOEDEL, "The construction of finite-difference approximations to ordinary differential equations," SIAM J. Numer. Anal., v. 15, 1978, pp. 450-465.

12. E. P. Doolan, J. J. H. Miller \& W. H. A. SChilders, Uniform Numerical Methods for Problems with Initial and Boundary Layers, Boole Press, Dublin, 1980.

13. K. V. EMEL'JANOV, "A truncated difference scheme for a linear singularly perturbed boundary value problem,” Soviet Math. Dokl., v. 25, 1982, pp. 168-172.

14. J. E. Flaherty \& W. Mathon, "Collocation with polynomial and tension splines for singularly perturbed boundary value problems,” SIAM J. Sci. Statist. Comput., v. 1, 1980, pp. 260-289.

15. J. E. FlaherTy \& R. E. O’Malley JR., "The numerical solution of boundary value problems for stiff differential equations," Math. Comp., v. 31, 1977. pp. 66-93.

16. J. E. Flaherty \& R. E. O’Malley, JR., "Numerical methods for stiff systems of two-point boundary value problems," SIAM J. Sci. Statist. Comput., v. 5, 1984, pp. 865-886.

17. R. B. Kellogg \& A. TSAN, "Analysis of some difference approximations for a singular perturbation problem without turning points," Math. Comp., v. 32, 1978, pp. 1025-1039.

18. B. KreISS \& H.-O. KREISS, "Numerical methods for singular perturbation problems," SIAM J. Numer. Anal., v. 18, 1981, pp. 262-276.

19. H.-O. KREISS, "Difference methods for stiff ordinary differential equations," SIAM J. Numer. Anal., v. 15, 1978, pp. 21-58.

20. S. H. Leventhal, "An operator compact implicit method of exponential type," J. Comput. Phys., v. 46,1982 , pp. $138-165$.

21. R. E. LYNCH \& J. R. Rice, "A high-order difference method for differential equations," Math. Comp., v. 34, 1980, pp. 333-372.

22. J. J. H. Miller (Editor), Boundary and Interior Lavers-Computational and Asymptotic Methods, Proc. BAIL I Conf., Boole Press, Dublin, 1980

23. J. J. H. Miller (Editor), Computational and Asymptotic Methods for Boundary and Interior Layers, Proc. BAIL II Conf., Boole Press, Dublin, 1982.

24. J. J. H. Miller (Editor), Proc. BAIl IIl Conf., Boole Press, Dublin, 1984.

25. K. NiedERDRENK \& H. YSERerstant, "Die gleichmässige Stabilität singulär gestörter diskreter und kontinuierlicher Randwertprobleme," Numer. Math., v. 41, 1983, pp. 223-253.

26. K. Nirima, "A uniformly convergent difference scheme for a semilinear singular perturbation problem,” Numer. Math., v. 43, 1984, pp. 175-198.

27. R. O. O’Mal.ley, JR., Introduction to Singular Perturbations, Academic Press, New York, 1974.

28. E. O'Riordan, "Singularly perturbed finite-element methods," Numer. Math., v. 44, 1984, pp. 425-434

29. E. O'RIORDAN \& M. STYNES, A Finite Element Method for a Singularly Perturbed Boundary Value Problem in Conservative Form, Proc. BAIL III Conf. (J. J. H. Miller, ed.), Boole Press, Dublin, 1984.

30. S. OSHER, "Nonlinear singular perturbation problems and one-sided difference schemes," SIAM J. Numer. Anal., v. 18, 1981, pp. 129-144.

31. J. R. Rice, The Approximation of Functions, Vol. 2, Nonlinear and Multivariate Theory, AddisonWesley, Reading, Mass., 1969.

32. D. R. SмIтн, "The multivariable method in singular perturbation analysis," SIAM Rev., v. 17. 1975, pp. 221-273.

33. M. VAN Veldhuizen, "High-order methods for a singularly perturbed problem," Numer. Math., v. 30,1978 , pp. $267-279$.

34. R. Weiss, "An analysis of the box and trapezoidal schemes for linear singularly perturbed boundary value problems," Math. Comp., v. 42, 1984, pp. 41-67. 


\title{
Supplement to \\ Uniform High-Order Difference Schemes for a Singularly Perturbed Two-Point \\ Boundary Value Problem
}

\author{
By Eugene C. Gartland, Jr.
}

\section{Appendix}

Proof of Theorem 3.1. For simplicity, we consider the local system on an interval $[-h, h]$ (suppress the subscript $i$ ) and define

$$
E(x) \equiv \exp \left(\frac{1}{\varepsilon} \int_{0}^{x} a\right)
$$

The auxiliary evaluation points are assumed to be given by

(A.1)

$$
\xi_{1}=0, \quad p=1,
$$

$$
\xi_{j}=-h+\frac{j-1}{p-1} h, \quad j=1, \ldots, 2 p-1, p=2,3 \ldots
$$

Ordering and scaling the conditions of exactness on $\left\{1, x, \ldots, x^{p}, E(x)\right.$, $\left.x E(x), \ldots, x^{p-1} E(x)\right\}$ appropriately gives rise to the local system (with $\left[\equiv \sum_{j=1}^{2 p-1}\right.$ )

$$
\begin{array}{lll}
\alpha_{-1}+\alpha_{0}+\alpha_{1} & -\left[\beta_{j} b\left(\varepsilon_{, j}\right)\right. & =0 \\
-\alpha_{-1}++\alpha_{1} & -\frac{1}{h}\left[\beta_{j}\left[\left(a\left(\xi_{j}\right)-a_{0}\right)+b\left(\xi_{j}\right) \xi_{j}\right]\right. & =\frac{a_{0}}{h} \\
\left.\left.E(-h) \alpha_{-1}+\alpha_{0}+E(h)\right)_{(1}\right] & -\left[\beta_{j} E\left(\varepsilon_{j}\right)\left(b-a^{\prime}\right)\left(\varepsilon_{j}\right)\right. & =0
\end{array}
$$

(A.2)

$$
\begin{gathered}
\frac{(-1)^{k}}{k} h \alpha_{-1}++\frac{1}{k} h_{\alpha_{1}}-\left[B_{j}\left\{\left[\frac{-\varepsilon(k-1)+a\left(\xi_{j}\right) \xi_{j}}{h}\right]\left(\frac{\xi_{j}}{h}\right)^{k-2}+O(h)\right\}=0,\right. \\
k=2, \ldots, p, \\
\frac{(-1)^{8}}{\ell} h E(-h)_{-1}++\frac{1}{i} h E(h) \alpha_{1}-\left[B_{j} E\left(i_{j}\right)\left\{\left[\frac{-\varepsilon(\ell-1)-a\left(\xi_{j}\right) \xi_{j}}{h}\right]\left(\frac{\xi_{j}}{h}\right)^{\ell-2}+O(h)\right\}=0,\right. \\
\ell=1, \ldots, p-1,
\end{gathered}
$$$$
\begin{aligned}
& \hat{\beta}_{j} \\
& \hat{s}_{j}
\end{aligned}
$$

where $a_{0}=a(0)$. 


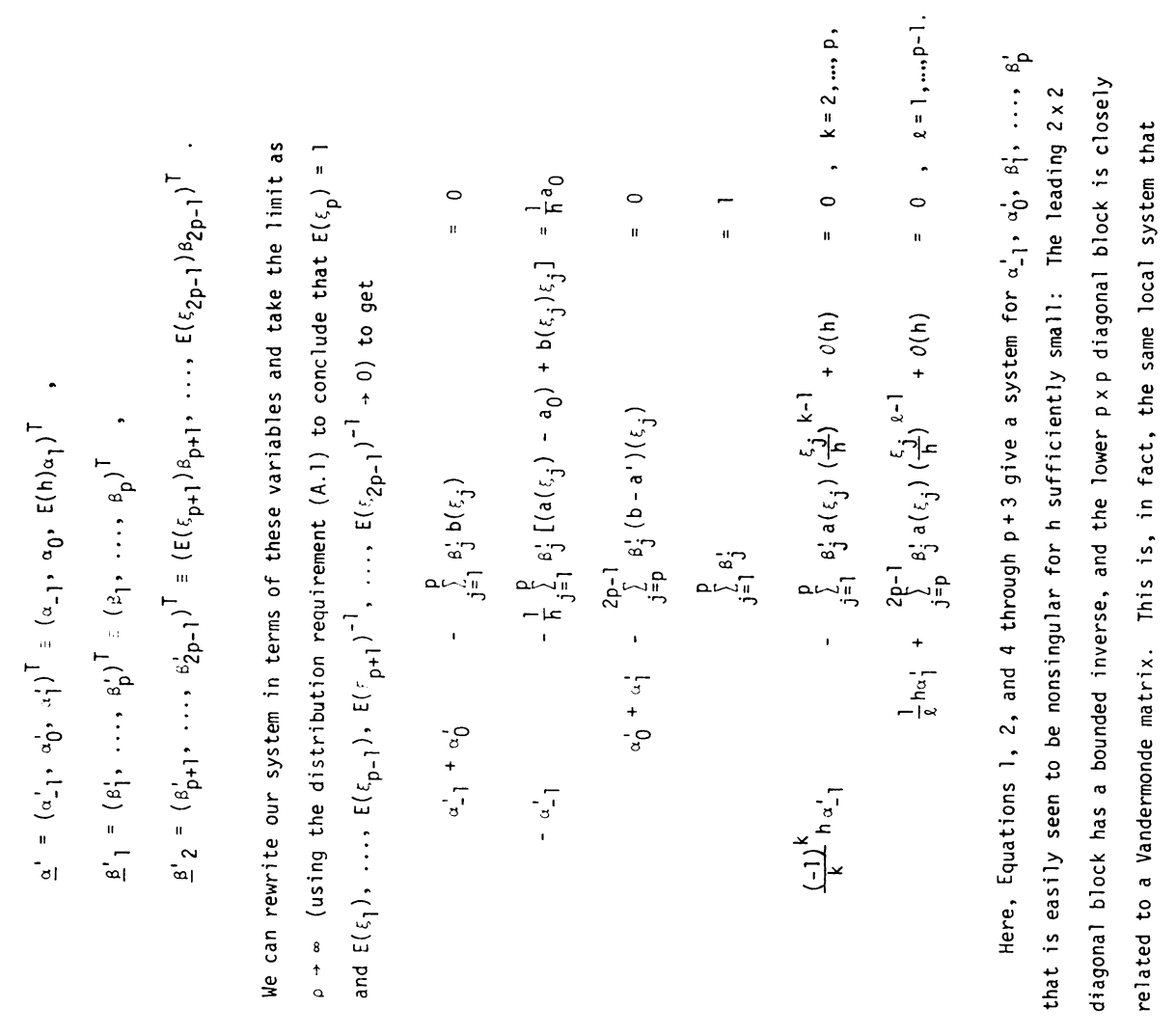

$\frac{T}{n}$

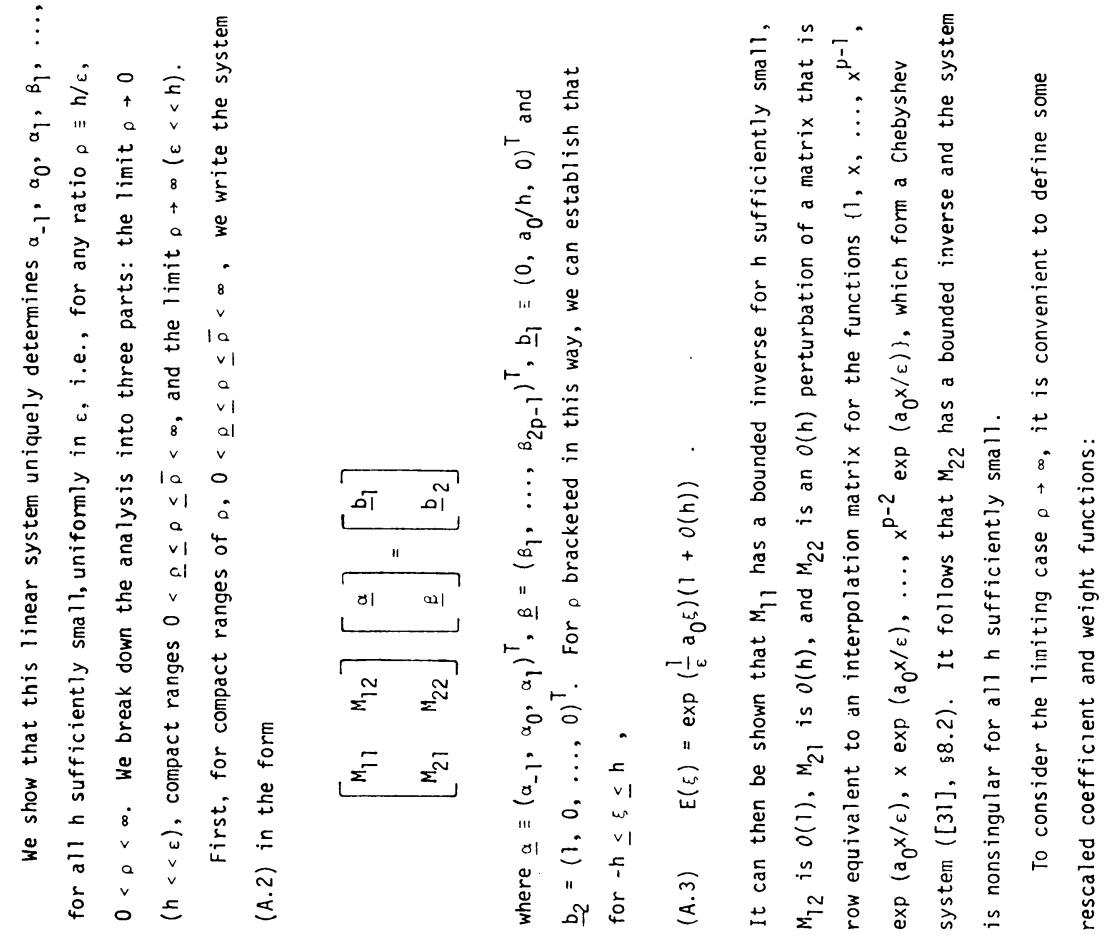




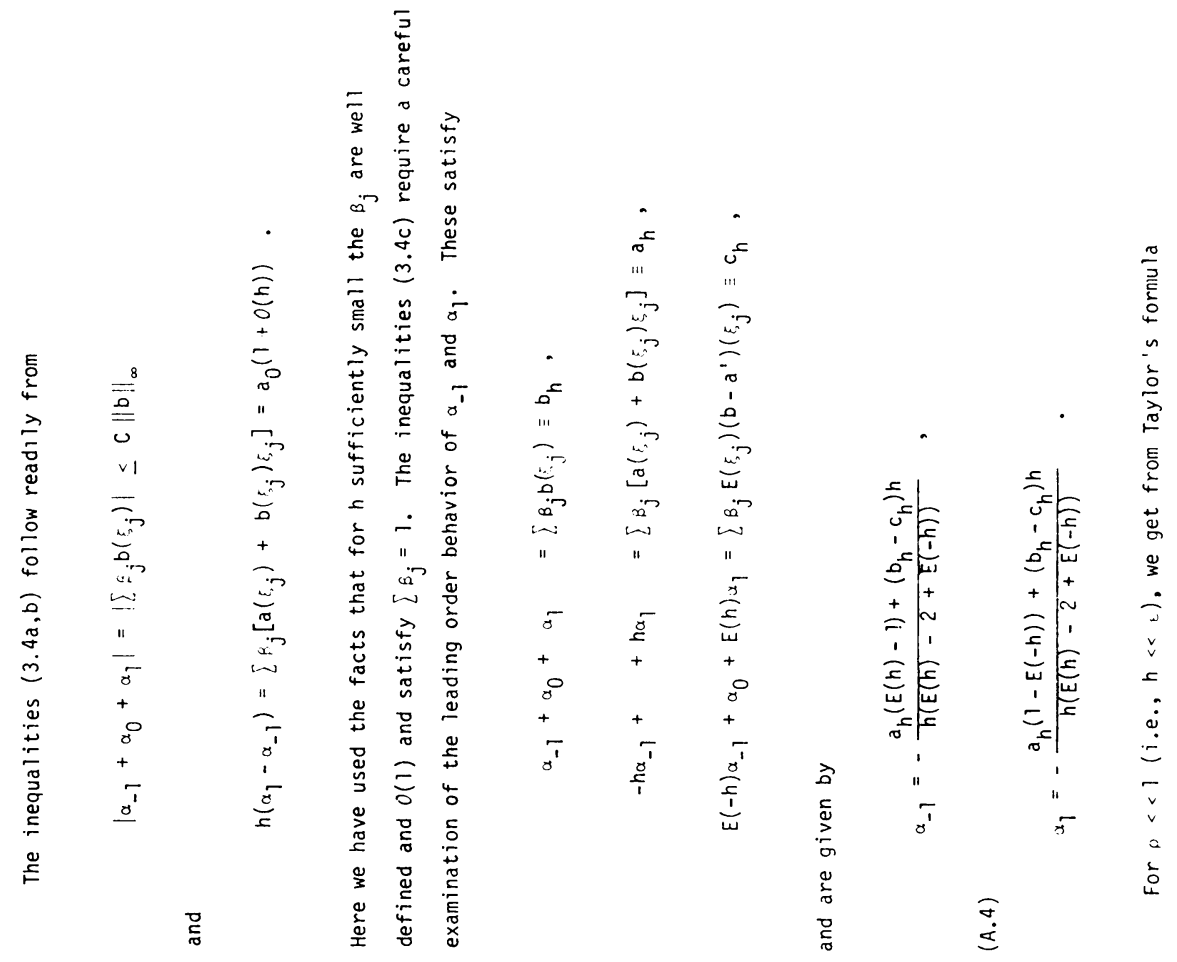

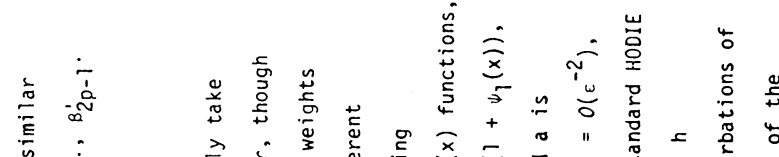

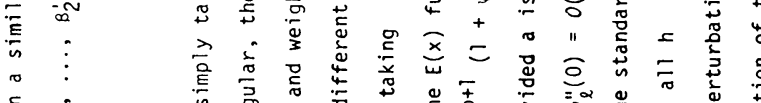

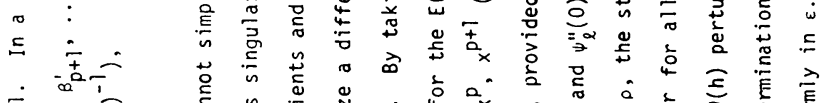

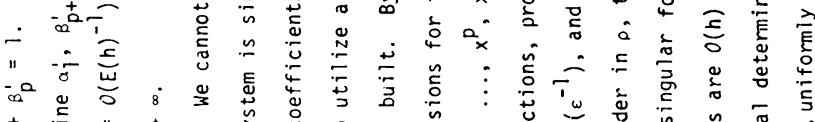

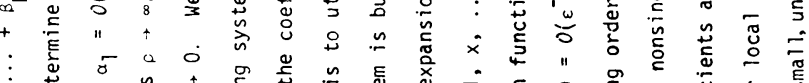
L

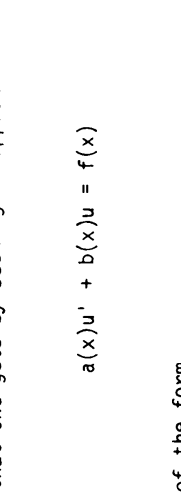

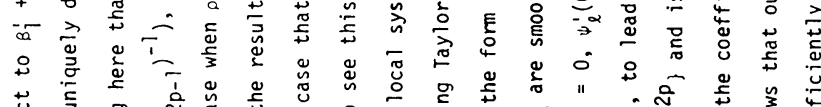

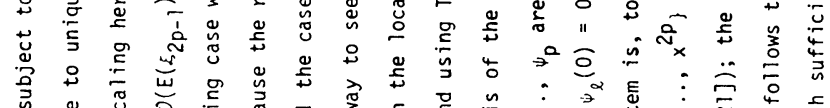

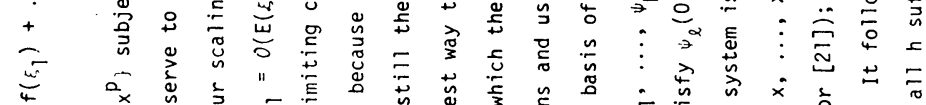

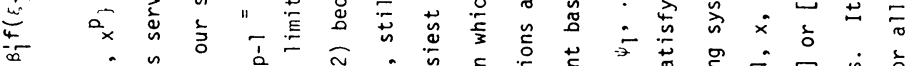

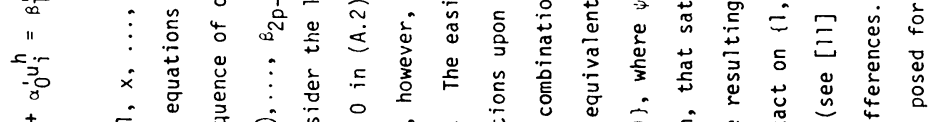

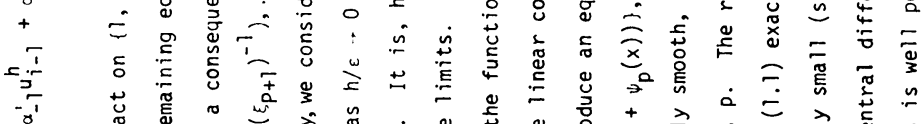

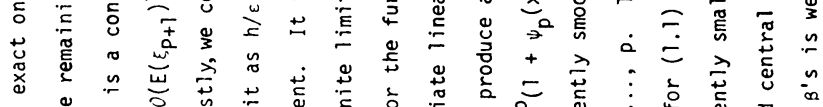

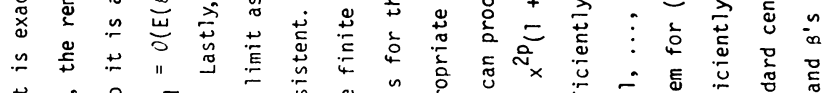

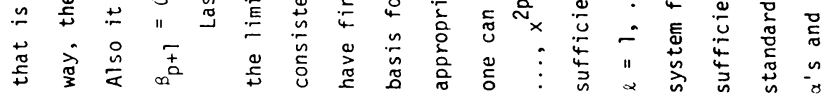



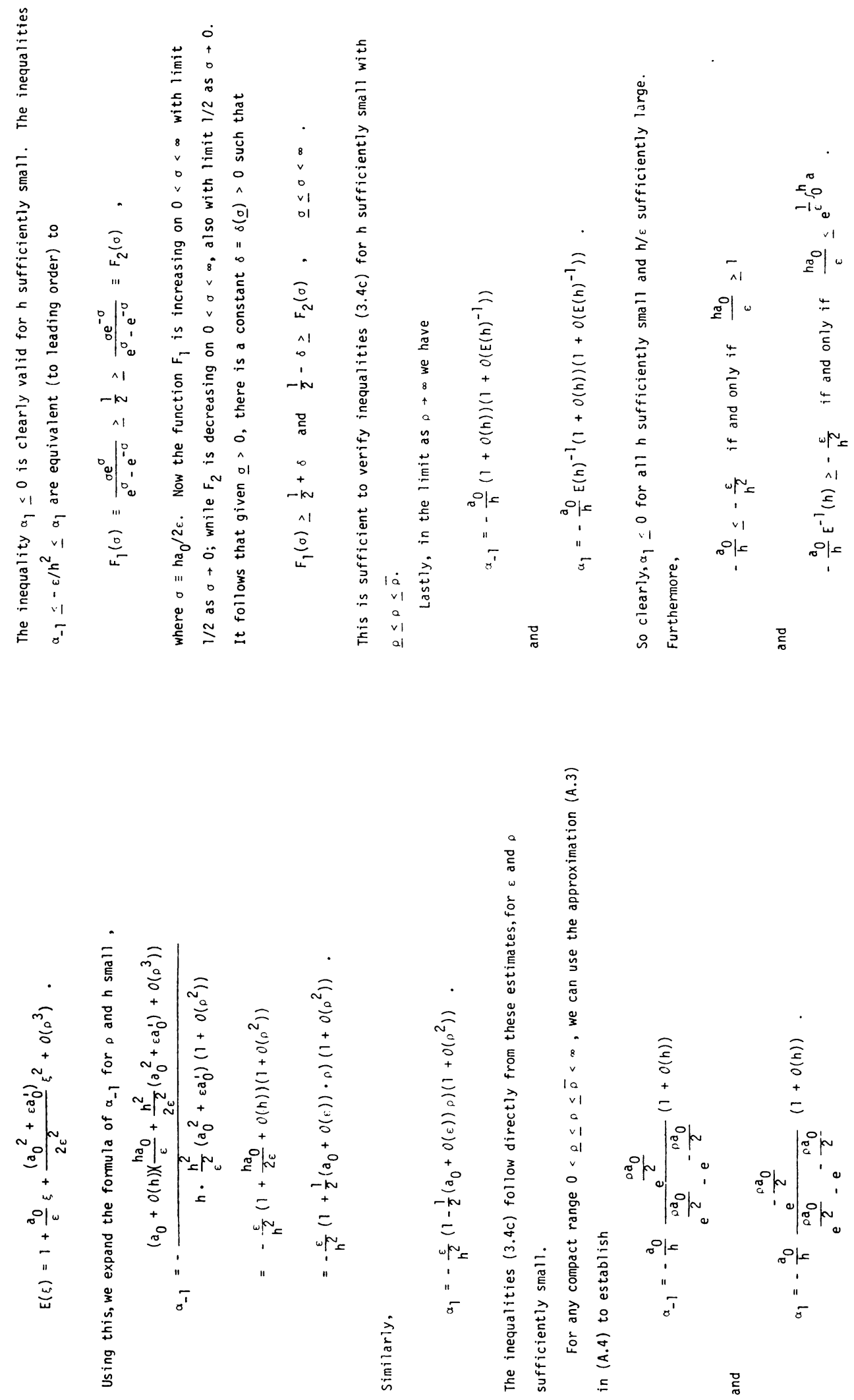


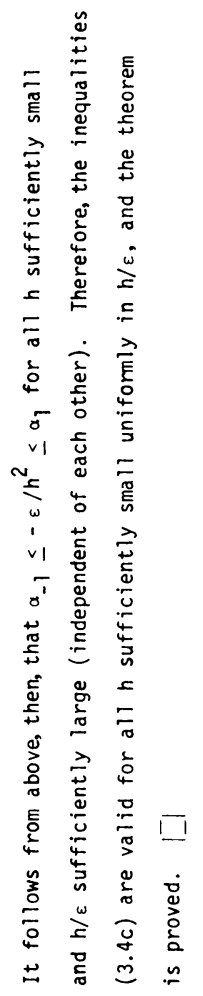

